\title{
Effective Modulus of Polycrystalline Aggregates in Different Geometrical Configurations
}

\author{
H. Fan, X. M. Xie* \\ School of Mechanical and Aerospace Engineering \\ Nanyang Technological University, Singapore 639798 \\ K.Y. Sze \\ Department of Mechanical Engineering \\ Hong Kong University, Hong Kong, P.R. China
}

\begin{abstract}
In the present study, a finite element scheme with random distribution strategy is employed to systematically investigate the modulus difference of polycrystalline copper aggregates in different geometrical configurations (three-dimensional bulk and thin film configurations). Firstly, the finite element simulation is performed to estimate the effective elastic constants in three-dimensional bulk configuration. The numerical estimations are in good agreement with the existing analytical solutions and experimental measurements. Secondly, the proven finite element scheme is extended to the prediction of the effective moduli of the free-standing and substrate-attached thin films. For the free-standing thin film, the effective Young's modulus decreases with reducing the film thickness. For the substrate-attached thin film, its effective modulus is affected by the relative stiffness between the substrate and the film. The spread of the effective moduli in different configurations could be as large as $20 \%$.
\end{abstract}

Keywords: effective modulus, finite element analysis, thin film, polycrystalline material, statistics.

* Corresponding author: Tel: (65) 6790-6551; Fax: 6791-1859.

Email address: XIEX0006@ntu.edu.sg (X.M. Xie).

Published in: Materials Science and Engineering A: Structural Materials: Properties, Microstructures and Processing, 527(18-19), 5008-5017, 2010 


\section{Introduction}

Polycrystalline thin films have been broadly used in microelectronic and micro-electro-mechanical system (MEMS) devices. In order to ensure the reliability and improve the performance of these devices, it is of paramount significance to understand the mechanical properties of the thin films. It has been noticed that the mechanical properties of thin films are often different from those of the corresponding bulk materials [1-4].

On the one hand, numerous investigators made efforts in measuring the mechanical properties of thin films [5]. There are two common configurations in the tests, namely, the free-standing thin films [6-8] and the substrate-attached thin films [2, 9-11]. Volinsky and co-workers [12] reported that the elastic moduli of the polycrystalline copper in different geometrical configurations may deviate as much as $20 \%$ to $30 \%$. Huang and Spaepen [7] found that the elastic moduli of various free-standing metal films and multi-layers (e.g., $\mathrm{Ag}, \mathrm{Cu}$ and $\mathrm{Ag} / \mathrm{Cu}$ multi-layers) measured by micro-tensile testing method are about $20 \%$ below the values in the bulk configurations. However, some investigators claimed that there is no modulus difference between the free-standing thin films and the corresponding bulk materials [8, 13-14]. Furthermore, Saha and Nix [9] focused on the influence of the substrate on the mechanical properties of the substrate-attached thin films by using nano-indentation. Yu and Spaepen [11] revealed that a polyimide (Kapton) substrate-attached copper thin film exhibited lower Young's modulus in comparison with its bulk value.

On the other hand, from the theoretical side, various finite element analyses were conducted to estimate the mechanical properties of the thin films [15-20]. den Tooder et al. [16] and Choi [18] established the Voronoi cells to study the effective constants in plane stress and plane strain state, respectively. Zhang and Sun [20] developed a multi-scale scheme to study the mechanical properties of textured polycrystalline nano-films in plane stress state. However, for the substrate-attached thin film, numerical simulation is rarely found in the open literatures. 
Furthermore, a number of analytical models for predicting the effective constants of polycrystalline aggregates were proposed. The well-known theoretical methods include bounds method [21-24], Hill's average [21] and self-consistent scheme [25-28]. Two remarkable pairs of bounds, Voigt-Reuss bounds [21] and Hashin-Shtrikman bounds [22], have been often taken as benchmark for the predicted effective constants. Hill's average, regarded as a good approximation for the effective moduli of polycrystalline aggregates by Hill [21], refers to the arithmetic or geometrical mean of Voigt upper and Reuss lower bounds. Although many complicate theoretical models were developed later, the Hill's average is preferred by many researchers due to its easy calculation and accuracy [29-30]. Morris [26] showed that there is only a couple of percents difference between Hill's average and prediction made by self-consistent scheme. It should be mentioned that most of the analytical models were derived for the three-dimensional bulk configuration. They may not suitable for the thin film configurations.

The present study is aimed to systematically investigate the modulus difference for polycrystalline aggregates in different geometrical configurations, including three-dimensional bulk configuration, the free-standing thin film and the substrate-attached thin film, within the scope of continuum linear elastic theory. The inspiration stems from Hill's contribution [21]. Voigt model assumed uniform strain in all the grains, which can be imagined as a parallel connection of the grains; while Reuss model assumed uniform stress in all the grains, which can be imagined as a series connection of all the grains. It is obvious that the three-dimensional configuration is the situation between Voigt model and Reuss model. Hill [21] made average on Voigt and Reuss models, which provided a good approximation for the effective constants of polycrystalline materials. In the case of the free-standing thin film, grains subject to less constraint from the neighboring grains in comparison with the grains in the bulk configuration. Hence, the effective modulus of the free-standing thin film may fall inside the region between the lower bound and the equivalent value of the three-dimensional bulk material. For the substrate-attached thin film, the grains are 
forced to deform together with the substrate. This constraint is more or less similar to Voigt's assumption. We foresee that the effective modulus of the substrate-attached thin film may fall inside the range between the three-dimensional bulk value and the upper bound.

The topic of using numerical techniques to look into the mechanical behaviors of polycrystalline aggregates has always been the interest of many investigators. Most of time numerical simulations for polycrystalline materials were performed via finite element models in which the morphological and crystallographic characteristics of microstructure was emphasized [32-36]. Kumar [32] adopted Voronoi tessellation in modeling a unit cube of polycrystalline solid and estimated the effective moduli. Barbe and co-colleagues [33, 34] simulated the polycrystalline aggregates by three-dimensional Voronoi cells to study their elastoplastic behaviors. Bhandari et al. [36] reconstructed the three-dimensional microstructure by using the crystallographic orientation maps of consecutive serial sections. In the present paper, we do not attempt to establish a finite element model which reflects the real microstructure of polycrystalline aggregates. Rather, a simple finite element scheme with a statistical consideration is proposed to predict the effective elastic constants of polycrystalline materials in different configurations. The accuracy and feasibility of such scheme will be verified by comparing the numerical results with the existing analytical solutions for the polycrystalline aggregates in the three-dimensional bulk configuration.

In the following sections, a finite element simulation based on a commercial software package ANSYS [31] is conducted. Section 2 outlines the framework of the proposed finite element scheme. In Section 3, this scheme is carried out for simulating polycrystalline copper material in three-dimensional bulk configuration. Verification is discussed by comparing the numerical results with analytical solutions. Section 4 investigates the effective Young's modulus of copper thin films, i.e., the free-standing thin film and the substrate-attached thin film. 


\section{Finite element scheme and statistical consideration}

Since our primary interest is to study the effective modulus difference of the polycrystalline materials in the three-dimensional bulk configuration and the thin film configurations, the actual morphology of polycrystalline aggregates, such as variation of the grain shape and size, will not be modeled into details in order to simplify our simulation procedures. We focus on the random distribution of crystallographic orientation of the grains, while the grain size is treated as a characteristic dimension of the microstructure and assumed to be constant, and all the grains aggregating the polycrystalline material have the identical shape. It will be shown that numerical results with these simplifications are in good agreement with existing analytical prediction (Hill's average).

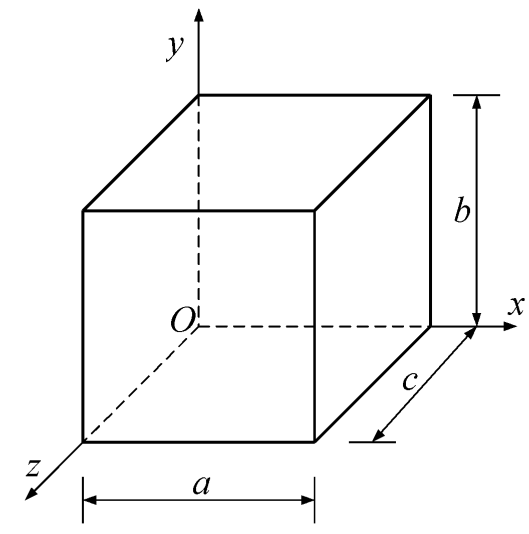

Figure 1. Schematic specimen with dimension $a \times b \times c$.

\subsection{Finite element scheme with random distribution of grain orientations}

In our numerical simulation, a prism specimen with macroscopic dimension $a \times b \times c$ is used to model a polycrystalline aggregate, shown in Fig.1. The edges are parallel to the global coordinate axes.

As the first step, the specimen is discretized into $N$ elements. Each element is considered as one grain. For a polycrystalline aggregate, each of the grains has random crystallographic orientation with respect to the global coordinate system. This implies that each grain possesses different material identity. Therefore, we need to generate $N$ orientations for the $N$ grains. Here, one grain orientation is defined by 
one set of Euler angles $(\alpha, \beta, \gamma)$. Considering the random distribution orientation of each grain in the specimen, we create $N$-sets of Euler angles in which each set is chosen randomly in $\alpha-\beta-\gamma$ space with uniform distribution. Taking advantage of tensorial transformation (see Appendix A), $N$-sets of elastic constants can be calculated based on $\mathrm{N}$-sets of Euler angles. Each set of the elastic constants is related to a unique material identity. These $N$-sets of the elastic constants are then input into the material sheets in the FEM package and $N$ types of materials are generated.

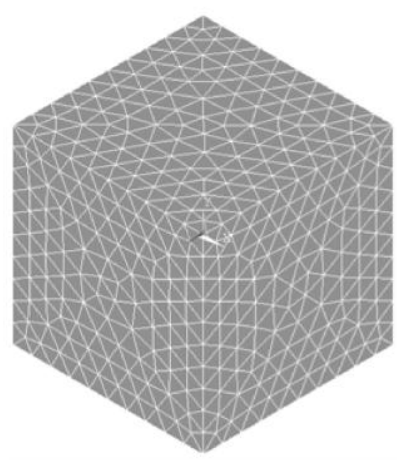

( a)

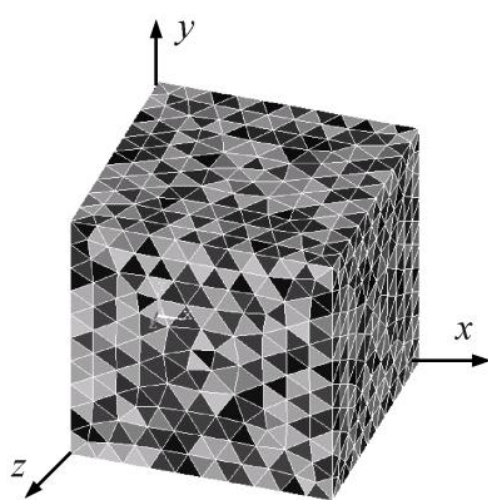

( b )

Figure 2. (a) The initial specimen model 1 by 1 by 1 is discretized into $N=7569$ tetrahedron-shaped elements with identical material properties. Each element is taken as one grain. (b) The established model consists of $N=7569$ tetrahedron-shaped grains. The crystallographic orientation for each grain is assigned randomly.

Fig. 2 shows the elements and grains in the specimen. The specimen is discretized by using the ten-node tetrahedral shape element type so that the shape of the grain is tetrahedron. Fig. 2 (a) depicts the specimen meshed into 7569 elements with identical material type. The constructed model for polycrystalline specimen consisting of 7569 grains with randomly assigned crystallographic orientation is presented in Fig. 2 (b). Different grey-levels represent the grains with various grains orientations. 
So far, each of the grains is modeled by only one element. Due to mismatched material properties among the grains, a large numerical error is expected if there is only one element in each grain. Therefore, a process of mesh refinement, which subdivides each grain into more elements, is needed. The mesh refinement not only gives the relaxation of stress concentration, but also smoothes the displacement variation between the grains with different material properties. Besides, the higher order element type is also adopted to improve the accuracy and accelerate the speed of convergence.

\subsection{Statistics consideration}

In the numerical simulation of the polycrystalline aggregates, our computational power is limited. The concept of statistics is therefore adopted. In the present work, the "population" refers to the specimen with a given number of the grains. For each "population", a sample consisting of thirty different finite element models is used to infer the effective elastic constants of the population. Because the crystallographic orientation for each grain in the finite element model is randomly assigned, thirty different predictions for the effective elastic constants are obtained. Two important statistical data, namely the sample mean value $(\mu)$ and the sample standard deviation $(s)$, for the effective elastic constants are achieved. According to Central Limit Theorem in statistics [37], the effective elastic constants for the specimen should be within the interval

$$
\mu \pm 1.96 \frac{s}{\sqrt{n}}
$$

with $95 \%$ confidence. The symbol $n$ is the sample size and equal to 30 in our work. Apparently, the interval can be narrowed by either decreasing the sample standard deviation or increasing the sample size. Keeping the statistical consideration in mind, we may achieve reliable accuracy for the predicted values by using the specimen with limited grain number and limited sample size. 


\section{Simulation on three-dimensional bulk configuration}

For illustration, polycrystalline copper material is taken as an example here for estimating the effective elastic constants in different geometrical configurations via the proposed finite element scheme. The elastic properties for the single crystal copper are given in Table 1 .

Table 1 Elastic constants for polycrystalline copper material. The anisotropic ratio is defined as $A R=2 C_{44} /\left(C_{11}-C_{12}\right)=2\left(S_{11}-S_{12}\right) / S_{44}$.

\begin{tabular}{cccccccc}
\hline Material type & $\begin{array}{c}C_{11} \\
(\mathrm{GPa})\end{array}$ & $\begin{array}{c}C_{44} \\
(\mathrm{GPa})\end{array}$ & $\begin{array}{c}C_{12} \\
(\mathrm{GPa})\end{array}$ & $\begin{array}{c}10^{3} S_{11} \\
(\mathrm{GPa})^{-1}\end{array}$ & $\begin{array}{c}10^{3} S_{44} \\
(\mathrm{GPa})^{-1}\end{array}$ & $\begin{array}{c}10^{3} S_{12} \\
(\mathrm{GPa})^{-1}\end{array}$ & $\begin{array}{c}\text { Anisotropic } \\
\text { ratio }\end{array}$ \\
\hline Copper & 168.4 & 75.4 & 121.4 & 15.00 & 13.26 & -6.28 & 3.21 \\
\hline
\end{tabular}

As discussed in the previous section, a prism specimen is used to model polycrystalline aggregates in bulk configuration. Since the grain size is a characteristic dimension with constant value, it is easy to construct specimen with diverse number of the grains through changing the specimen's dimensions $a, b$ and $c$. The ten-node tetrahedral shape element is employed to discretize the specimen. Each of the grains is randomly assigned one crystallographic orientation. At this stage, each grain is modeled by only one element so that the above mesh setup is termed as the zero-th level of mesh refinement (R0). To reduce the computational error, the first (R1), second (R2), third (R3) and fourth (R4) levels of mesh refinement are also performed in which each grain is subdivided into $2^{3}(=8), 3^{3}(=27), 4^{3}(=64)$ and $8^{3}(=512)$ elements, respectively.

\subsection{Boundary and loading conditions}

To deform the specimen, the following boundary and loading conditions are imposed.

$$
\begin{array}{ll}
u_{x}=0 & \text { at } x=0 \\
u_{x}=\Delta & \text { at } x=a
\end{array}
$$




$$
\begin{array}{ll}
u_{y}=0, & \text { along } x=0, y=0 \\
u_{z}=0, & \text { along } x=0, z=0
\end{array}
$$

where $\Delta$ is the prescribed displacement. The symbols, $u_{x}, u_{y}$ and $u_{z}$, denote the displacement along the directions $x, y$ and $z$ in the global coordinate system respectively. In addition to the aforementioned boundary conditions, the non-specified boundary conditions are in "traction-free" condition.

Based on the principle of energy balance, the effective Young's modulus can be calculated by

$$
E=\frac{2 a U}{\Delta^{2} A}
$$

where $a$ is the length of edge along the $x$ direction, illustrated in Fig. 1; $A$ is the cross sectional area of the plane perpendicular to axis $x$; $U$ is the total strain energy collected from all the elements in the specimen.

For the Poisson's ratio, we have

$$
\begin{aligned}
& v_{x y}=-\frac{\varepsilon_{y}}{\varepsilon_{x}}=-\frac{\left[\bar{u}_{y}(y=b)-\bar{u}_{y}(y=0)\right] / b}{\Delta / a} \\
& v_{x z}=-\frac{\varepsilon_{z}}{\varepsilon_{x}}=-\frac{\left[\bar{u}_{z}(z=c)-\bar{u}_{z}(z=0)\right] / c}{\Delta / a}
\end{aligned}
$$

where $\bar{u}_{y}(y=b)$ and $\bar{u}_{y}(y=0)$ are the averaged nodal displacement in the $y$ direction over the specified surfaces $y=b$ and $y=0$ of the specimen, respectively, $\bar{u}_{z}(z=c)$ and $\bar{u}_{z}(z=0)$ are the averaged nodal displacement in the $z$ direction over the specified surfaces $z=c$ and $z=0$ of the specimen, respectively. 
Table 2 Summary of finite element simulation for copper bulk material. The statistic mean value and standard deviation (S.D.) for each specimen at each level of mesh refinement are obtained from a sample size of $n=30$.

\begin{tabular}{|c|c|c|c|c|c|c|c|c|c|c|c|}
\hline \multirow{3}{*}{$\begin{array}{l}\text { Specimen } \\
\text { size }\end{array}$} & \multirow{3}{*}{$\begin{array}{l}\text { Element } \\
\text { shape }\end{array}$} & \multirow{3}{*}{$\begin{array}{l}\text { Number } \\
\text { of } \\
\text { grains }\end{array}$} & \multirow{3}{*}{$\begin{array}{l}\text { Refine- } \\
\text { ment } \\
\text { level }\end{array}$} & \multirow{3}{*}{$\begin{array}{l}\text { Number } \\
\text { of } \\
\text { elements }\end{array}$} & \multirow{3}{*}{$\begin{array}{l}\text { Number } \\
\text { of } \\
\text { nodes }\end{array}$} & \multirow{2}{*}{\multicolumn{2}{|c|}{$\begin{array}{c}\text { Effective } \\
\text { Young's } \\
\text { modulus (GPa) }\end{array}$}} & \multicolumn{4}{|c|}{ Effective Poisson's ratio } \\
\hline & & & & & & & & \multicolumn{2}{|c|}{$v_{x y}$} & \multicolumn{2}{|c|}{$v_{x z}$} \\
\hline & & & & & & Mean & S.D. & Mean & S.D. & Mean & S.D. \\
\hline \multirow{2}{*}{$1 \times 1 \times 1$} & Tetrahedron & \multirow{2}{*}{7569} & R0 & 7569 & 11554 & 135.48 & 0.182 & 0.3356 & 0.001 & 0.3353 & 0.001 \\
\hline & (Solid187) & & $\mathrm{R} 1$ & 60539 & 86310 & 131.09 & 0.182 & 0.3409 & 0.001 & 0.3406 & 0.001 \\
\hline \multirow{3}{*}{$1 \times 1 \times 0.5$} & \multirow{3}{*}{$\begin{array}{l}\text { Tetrahedron } \\
\text { (Solid187) }\end{array}$} & \multirow{3}{*}{4140} & R0 & 4140 & 6533 & 135.12 & 0.422 & 0.3355 & 0.002 & 0.3361 & 0.002 \\
\hline & & & $\mathrm{R} 1$ & 33106 & 48019 & 130.73 & 0.404 & 0.3406 & 0.002 & 0.3415 & 0.002 \\
\hline & & & $\mathrm{R} 2$ & 111750 & 157591 & 129.43 & 0.422 & 0.3421 & 0.002 & 0.3431 & 0.002 \\
\hline \multirow{4}{*}{$0.5 \times 0.5 \times 0.5$} & \multirow{4}{*}{$\begin{array}{c}\text { Tetrahedron } \\
\text { (Solid187) }\end{array}$} & \multirow{4}{*}{809} & R0 & 809 & 1464 & 134.67 & 1.014 & 0.3374 & 0.006 & 0.3365 & 0.005 \\
\hline & & & $\mathrm{R} 1$ & 6469 & 10092 & 130.50 & 1.002 & 0.3425 & 0.006 & 0.3411 & 0.005 \\
\hline & & & $\mathrm{R} 2$ & 21829 & 32352 & 129.14 & 1.018 & 0.3439 & 0.006 & 0.3427 & 0.005 \\
\hline & & & R3 & 51722 & 74695 & 128.64 & 1.025 & 0.3445 & 0.006 & 0.3432 & 0.005 \\
\hline \multirow{5}{*}{$0.3 \times 0.3 \times 0.3$} & \multirow{5}{*}{$\begin{array}{c}\text { Tetrahedron } \\
\text { (Solid187) }\end{array}$} & \multirow{5}{*}{342} & R0 & 342 & 634 & 134.39 & 1.701 & 0.3400 & 0.009 & 0.3340 & 0.009 \\
\hline & & & $\mathrm{R} 1$ & 2370 & 4305 & 130.12 & 1.637 & 0.3445 & 0.009 & 0.3394 & 0.009 \\
\hline & & & $\mathrm{R} 2$ & 9225 & 13759 & 128.82 & 1.684 & 0.3461 & 0.009 & 0.3407 & 0.009 \\
\hline & & & R3 & 21852 & 31705 & 128.31 & 1.701 & 0.3467 & 0.009 & 0.3412 & 0.009 \\
\hline & & & R4 & 174825 & 243234 & 127.63 & 1.693 & 0.3474 & 0.009 & 0.3420 & 0.009 \\
\hline \multirow{3}{*}{$0.9 \times 0.9 \times 0.9$} & \multirow{3}{*}{$\begin{array}{c}\text { Hexahedron } \\
\text { (Solid186) }\end{array}$} & \multirow{3}{*}{729} & R0 & 729 & 3700 & 129.1 & 0.994 & 0.3447 & 0.005 & 0.3439 & 0.006 \\
\hline & & & $\mathrm{R} 1$ & 5832 & 26353 & 127.46 & 0.999 & 0.3460 & 0.005 & 0.3449 & 0.006 \\
\hline & & & R2 & 19683 & 85456 & 127.14 & 1.000 & 0.3463 & 0.005 & 0.3451 & 0.005 \\
\hline
\end{tabular}
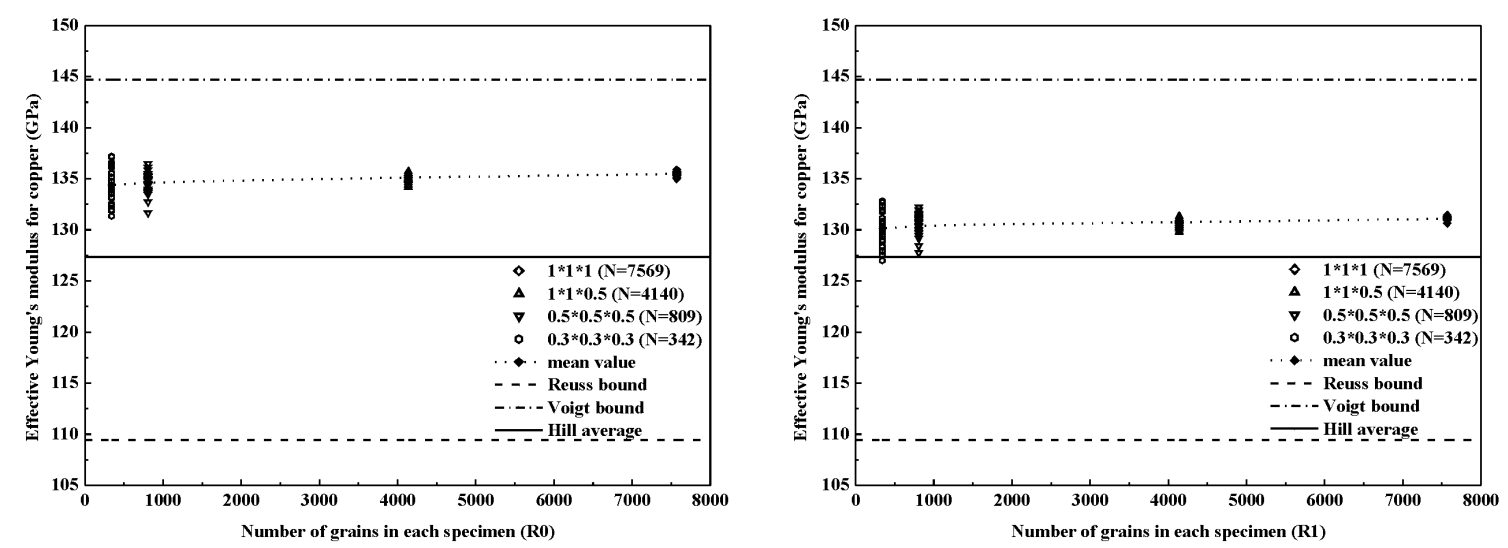

Figure 3. Scatter of the effective Young' s modulus for the specimen with different number of the grains in the zeroth (R0) and the first (R1) levels of mesh refinement for polycrystalline copper material respectively. In R0 level, each of the grains has only one element, while in R1 level, eight elements are included in each of the grains. 


\subsection{Numerical results and discussion}

The numerical simulation is carried out for each specimen in different levels of mesh refinement with sample size $n=30$. The raw data are summarized in Table 2 . Fig. 3 shows the scatter of the effective Young's modulus of the copper bulk material versus the number of the grains in the zero-th and the first levels of mesh refinement, respectively. Also included in the figure is Voigt-Reuss bounds and Hill's average for comparison (see Appendix B). It is seen that the mean value of the effective Young's modulus varies slightly when the number of the grains increases from 342 (specimen with dimension $0.3 \times 0.3 \times 0.3$ ) to 7569 (specimen with dimension $1 \times 1 \times 1$ ), whereas the corresponding standard deviation decreases dramatically. All the numerical predictions fall in between the lower and upper bounds.

Due to the limitation of our computer power and time cost, larger number of the grains and higher level of mesh refinement cannot be adopted simultaneously. To explore the trend of increasing the mesh refinement level, the specimen with 342 grains is analyzed at different mesh levels. Fig. 4 gives the mean value and variance of the effective stiffness from the zero-th (R0) to the fourth (R4) level of mesh refinement. It shows that the effective Young's modulus reduces and approaches to the Hill's average value with increasing the mesh refinement level.

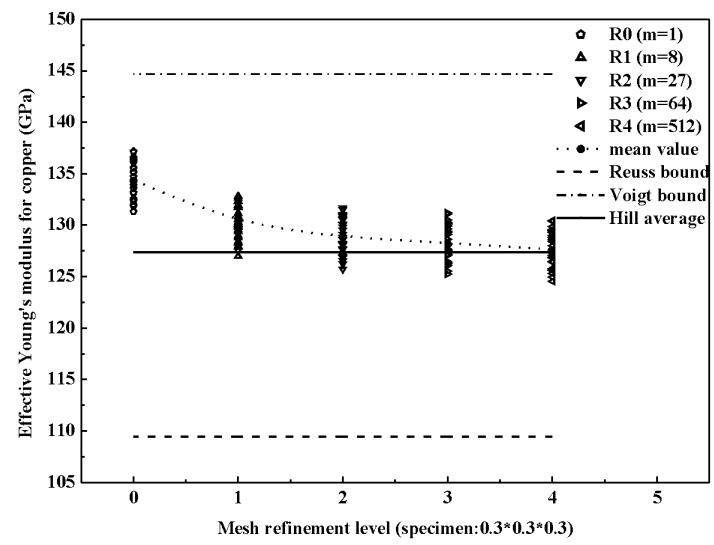

Figure 4. Scatter of the effective Young' s modulus for the specimen $0.3 \times 0.3 \times 0.3$ from the zeroth $(\mathrm{R} 0)$ to the fourth (R4) level of mesh refinement. In different levels of mesh refinement, each of the grains includes certain number of the elements denoted by $\mathrm{m}$. 
Based on these numerical insights, two trends can be identified clearly. First, it is seen that the larger the number of the grains is, the lower the standard deviation is. Second, the higher the level of mesh refinement or the larger the number of the elements in one grain is, the lower the mean value of the effective stiffness is. The first trend is in agreement with statistics in common sense. The second trend is in line with the finite element theory for the displacement-based formation. Comparing the predicted value in the second mesh level with that obtained in the fourth level of mesh refinement, there is only $1 \%$ difference of the effective stiffness at the cost of 20 -fold increase of the total number of elements. Hence, in combination with statistical consideration, we have confidence that the present numerical simulation with limited number of grains (below 1000 grains) and elements (in the second mesh refinement level, R2) can achieve reliable accuracy.

So far, the shape of the grains aggregating the specimen is tetrahedral. To shed some light on the influence of the grain shape on the effective elastic constants, another grain shape, namely cube or hexahedron, is used. A specimen with 729 hexahedron-shaped grains is constructed, as shown in Fig. 5. Each of the grains has randomly assigned crystallographic orientation and contains only one element. Twenty-node hexahedral type element is chosen to discretize the specimen. The corresponding numerical results are listed in the Table 2. In comparison with the corresponding results for the specimen made up of comparable number of tetrahedron-shaped grains, less than one percent discrepancy is observed. It indicates that the grain shape affects the predicted effective stiffness slightly. Similar feature was reported by Kumar [32] and Nozaki and Taya [38].

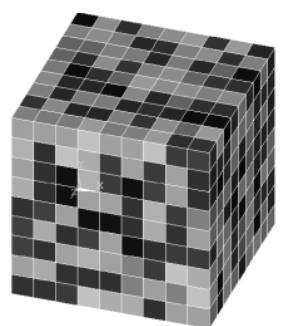

Figure 5. The specimen composed of 729 hexahedral grains. 
Table 3 The prediction value of the effective elastic Young's modulus and Poisson's ratio for polycrystalline copper aggregate by using different methods.

\begin{tabular}{ccc}
\hline Approach & $\begin{array}{c}\text { Effective Young's } \\
\text { modulus (GPa) }\end{array}$ & Poisson's ratio \\
\hline Experimental value [39] & $125-135$ & $0.34-0.35$ \\
Hill's average [21] & 127.36 & 0.345 \\
Reuss lower bound [21] & 109.44 & \\
Voigt upper bound [21] & 144.69 & 0.346 \\
Finite element simulation & 127.14 & \\
\hline
\end{tabular}

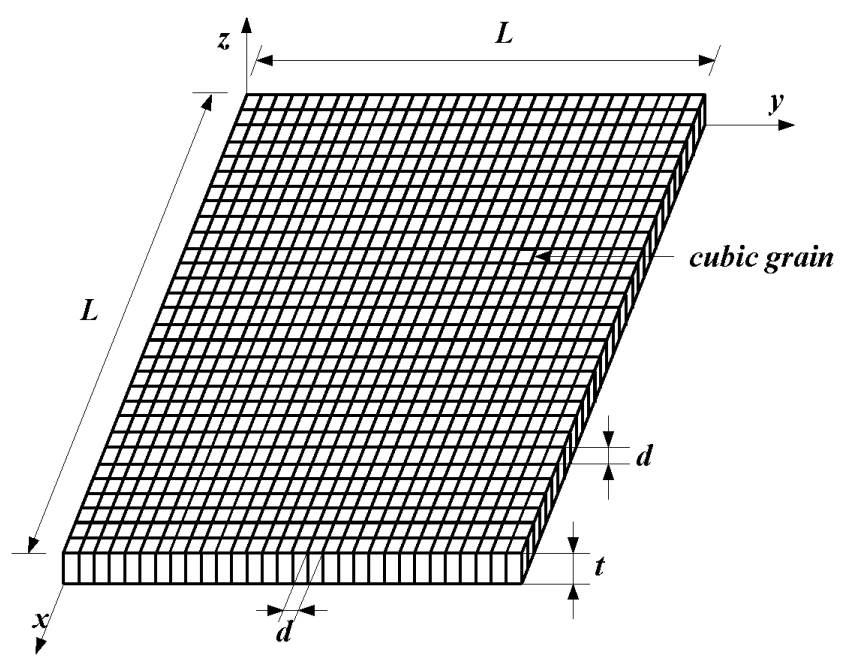

Figure 6. A free-standing thin film with only one grain along the thickness direction. The specimen is discretized into $(L / d) \times(L / d)$ grains with equal size $d \times d \times t . \quad L, \quad t$ and $d$ denote the specimen length, the film thickness and the grain size, respectively.

To verify the accuracy and feasibility of our finite element analysis, existing analytical solutions and experimental data are given in Table 3. It is noticed that the present finite element simulation provides accurate estimation for the polycrystalline copper aggregate. Moreover, the predicted value falls in between the lower and upper bounds. Hence, we are confident that the present finite element scheme can provide accurate effective elastic constants for the polycrystalline aggregates. In the next section, the verified finite element scheme is used to simulate the free-standing thin film and the substrate-attached thin film.

To make the numerical data complete, the statistical data for the predicted Poisson's ratio are also included in Table 2. 


\section{Simulation on thin film configurations}

The geometrical parameters of a thin film are illustrated schematically in Fig. 6 for its micro-structural and macro-structural dimensions. The parameter ' $d$ ', which is taken as the basic dimension, is the in-plane dimension of a grain. ' $t$ ' represents the thickness of the thin film, and ' $L$ ' is the macroscopic dimension of the thin film in $x-y$ plane. From now on, all the geometrical parameters except the grain size, i.e., $t, L$ and $h$ (the thickness of the substrate used in the next section), are normalized by the grain size as $t / d, L / d$ and $h / d$, respectively. The significant feature of the thin film's microstructure is that each of the grains "penetrating thru" the film thickness direction. In other words, the thin film has columnar grain structure. In our terminology, the "thin" film is defined by the dimensionless ratio ' $t / d$ ', rather by the absolute value of the thickness.

\subsection{Simulation on free-standing thin film}

The loading and boundary conditions are imposed as follows.

$$
\begin{array}{ll}
u_{x}=0 & \text { at } x=0 \\
u_{x}=\Delta & \text { at } x=L \\
u_{y}=0, & \text { along } x=0, y=0 \\
u_{z}=0, & \text { along } x=0, z=0
\end{array}
$$

where $\Delta$ is prescribed. The non-specified boundary conditions are traction free. The effective Young's modulus of the free-standing thin film can be extracted from the finite element numerical data as

$$
E=\frac{2 L U}{\Delta^{2} A}
$$

where $A$ is the cross sectional area of the specimen at $x=L$ and $U$ is the total strain energy collected from all the grains of the thin film. 


\subsubsection{Sensitivity of the effective Young's modulus to the film thickness}

The specimen with different ratio between the film thickness $(t)$ and the grain size (d) is constructed. The ratio, $t / d$, takes the value from 0.1 to 1 . Each specimen consists of 900 cubic grains with randomly assigned crystallographic orientation. In our simulations, the three-dimensional twenty-node hexahedral type element is used. Thirty numerical simulations are carried out for the specimen with given ratio ' $t / d$ '. Fig. 7 plots the mean value and dispersion of the effective stiffness for copper free-standing thin film in the function of the ratio ' $t / d$ ' after mesh refinement. The results given in Fig. 7 show the effective stiffness decreases with reducing the film thickness.

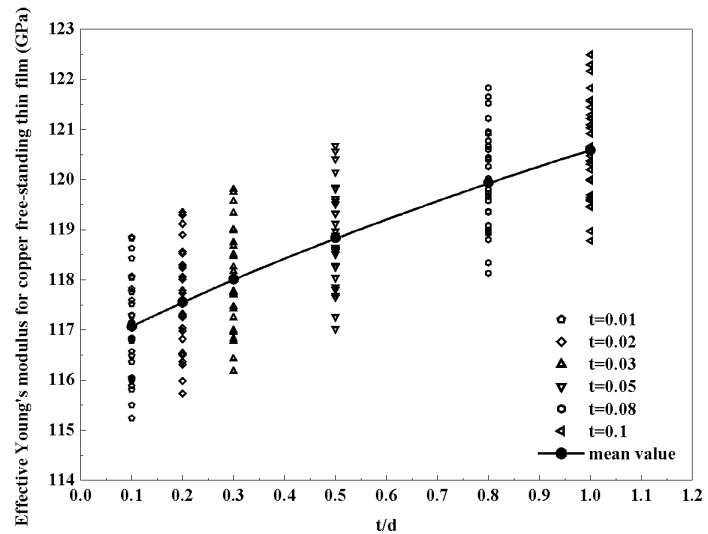

Figure 7.Scatter of the effective Young' s modulus for the copper freestanding thin film versus the ratio $t / d$. The specimen is composed of $30 \times 30$ grains with equal size $d \times d \times t$. Each grain is subdivided into 48 elements after mesh refinement.

\subsubsection{Plane stress approximation for the free-standing thin film}

When the film thickness is much less than the grain size, the free-standing thin film may be simplified from a three-dimensional thin film to a two-dimensional plane stress state. There have been a number of numerical simulations by using the plane stress approximation in the open literature, for example, den Tooder et al. [16], Choi [18], and Sze and Sheng [19]. The plane stress simulation is conducted for specimen with different number of the grains. It should be emphasized that the grain orientation is randomly assigned with three Euler angles and the plane stress condition $\left(\sigma_{z z}=\sigma_{y z}=\sigma_{x z}\right.$ 
$=0$ ) is incorporated into the material properties matrix with respect to global coordinate system.

Table 4 summarizes the statistical data for the plane stress approximation with various numbers of the grains and the levels of mesh refinement. When the number of the grains increases, the mean value of the effective stiffness has negligible variation and the corresponding standard deviation changes dramatically. Such feature is consistent with the observed phenomenon in Section 3.2. When the specimen is built up with 900 grains, the predicted stiffness in the plane stress approximation is very close to the corresponding value obtained from the free-standing thin film with ratio $t / d=0.1$. It is reasonable to believe that the effective stiffness obtained from the plane stress approximation is the limit of the stiffness for the free-standing thin film when the ratio $t / d$ is much less than 1 .

Table 4 Statistical data for the effective Young's modulus of copper material in plane stress approximation. The sample size is $n=30$.

\begin{tabular}{|c|c|c|c|c|c|c|}
\hline \multirow{2}{*}{$\begin{array}{l}\text { Specimen } \\
\text { size } \\
(L / d) \times(L / d)\end{array}$} & \multirow[b]{2}{*}{ Element type } & \multirow{2}{*}{$\begin{array}{l}\text { Number } \\
\text { of grains }\end{array}$} & \multirow{2}{*}{$\begin{array}{c}\text { Refinement } \\
\text { level }\end{array}$} & \multirow{2}{*}{$\begin{array}{c}\text { Number } \\
\text { of } \\
\text { elements } \\
\text { in each } \\
\text { grain }\end{array}$} & \multicolumn{2}{|c|}{$\begin{array}{c}\text { Effective } \\
\text { Young's modulus }\end{array}$} \\
\hline & & & & & $\begin{array}{l}\text { Mean } \\
(\mathrm{GPa})\end{array}$ & $\begin{array}{l}\text { S.D. } \\
(\mathrm{GPa})\end{array}$ \\
\hline \multirow{3}{*}{$20 \times 20$} & \multirow{3}{*}{$\begin{array}{c}\text { Quadrilateral } \\
\text { (Plane 183) }\end{array}$} & \multirow{3}{*}{400} & R0 & 1 & 117.05 & 1.600 \\
\hline & & & $\mathrm{R} 1$ & 9 & 116.56 & 1.577 \\
\hline & & & $\mathrm{R} 2$ & 81 & 116.49 & 1.576 \\
\hline \multirow{3}{*}{$30 \times 30$} & \multirow{3}{*}{$\begin{array}{c}\text { Quadrilateral } \\
\text { (Plane 183) }\end{array}$} & \multirow{3}{*}{900} & R0 & 1 & 116.98 & 1.063 \\
\hline & & & $\mathrm{R} 1$ & 9 & 116.50 & 1.061 \\
\hline & & & $\mathrm{R} 2$ & 81 & 116.42 & 1.060 \\
\hline \multirow{3}{*}{$40 \times 40$} & \multirow{3}{*}{$\begin{array}{c}\text { Quadrilateral } \\
\text { (Plane 183) }\end{array}$} & \multirow{3}{*}{1600} & R0 & 1 & 116.63 & 0.638 \\
\hline & & & $\mathrm{R} 1$ & 9 & 116.14 & 0.638 \\
\hline & & & $\mathrm{R} 2$ & 81 & 116.07 & 0.637 \\
\hline \multirow{3}{*}{$50 \times 50$} & \multirow{3}{*}{$\begin{array}{c}\text { Quadrilateral } \\
\text { (Plane 183) }\end{array}$} & \multirow{3}{*}{2500} & R0 & 1 & 116.92 & 0.539 \\
\hline & & & R1 & 9 & 116.43 & 0.542 \\
\hline & & & R2 & 81 & $\mathrm{n} / \mathrm{a}$ & $\mathrm{n} / \mathrm{a}$ \\
\hline \multirow{3}{*}{$60 \times 60$} & \multirow{3}{*}{$\begin{array}{c}\text { Quadrilateral } \\
\text { (Plane 183) }\end{array}$} & \multirow{3}{*}{3600} & R0 & 1 & 116.72 & 0.328 \\
\hline & & & R1 & 9 & 116.23 & 0.329 \\
\hline & & & R2 & 81 & $\mathrm{n} / \mathrm{a}$ & $\mathrm{n} / \mathrm{a}$ \\
\hline \multirow{3}{*}{$80 \times 80$} & \multirow{3}{*}{$\begin{array}{c}\text { Quadrilateral } \\
\text { (Plane 183) }\end{array}$} & \multirow{3}{*}{6400} & R0 & 1 & 116.81 & 0.213 \\
\hline & & & $\mathrm{R} 1$ & 9 & 116.32 & 0.211 \\
\hline & & & $\mathrm{R} 2$ & 81 & $\mathrm{n} / \mathrm{a}$ & n/a \\
\hline
\end{tabular}




\subsection{Simulation on the substrate-attached thin film}

\subsubsection{Boundary and loading conditions}

The geometric configuration and corresponding geometric parameters $(t, d, h$ and $L)$ of a substrate-attached thin film is plotted in Fig. 8. The thickness of the substrate is much larger than the thickness of the substrate-attached thin film.

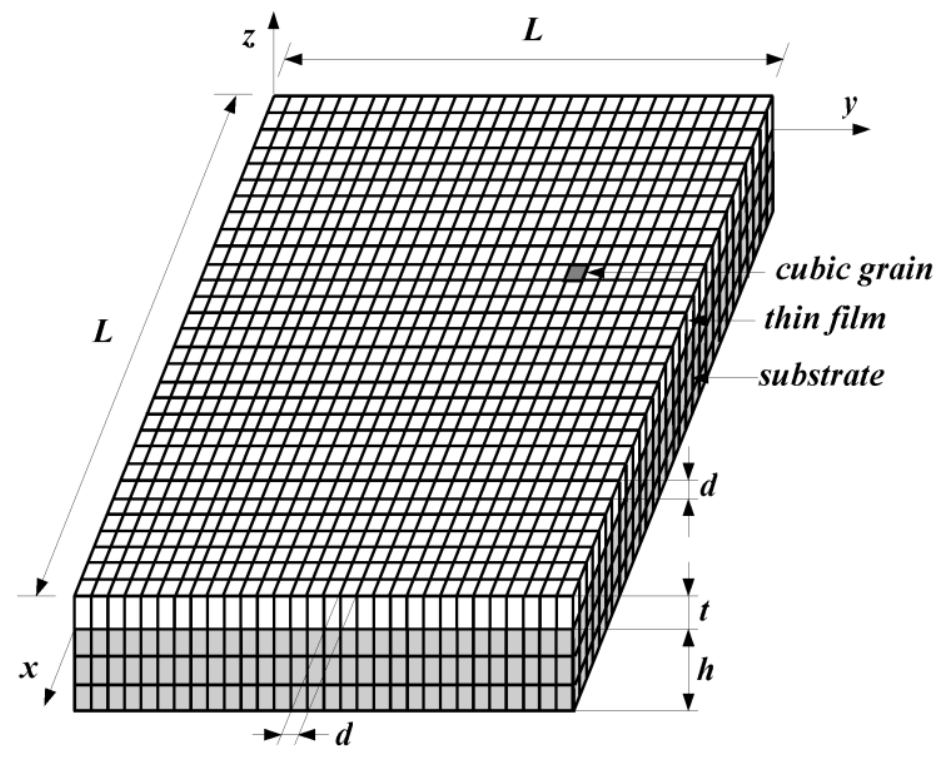

Figure 8. A substrate-attached thin film with only one grain in the thickness direction. The attached thin film is discretized into $(L / d) \times(L / d)$ grains with equal size $d \times d \times t . L, d, t$ and $h$ denote the length of specimen, the grain size, the thin film thickness and the substrate thickness, respectively.

The boundary and loading conditions are prescribed on the film/substrate structure as

$$
\begin{array}{ll}
u_{x}=0 & \text { at } x=0 \\
u_{x}=\Delta & \text { at } x=L \\
u_{y}=0 & \text { along } x=0, y=0 \\
u_{z}=0 & \text { along } x=0, z=-h
\end{array}
$$

where ' $h$ ' denotes the substrate thickness. The other non-specified boundary conditions are traction free. The effective Young's modulus of the substrate-attached thin film is also evaluated based on Eq. (7). It should be mentioned that the strain 
energy, $U$, is the summation of the strain energy stored in all the grains made up of the attached thin film.

\subsubsection{Sensitivity of the effective Young's modulus to the substrate}

In order to compare with the numerical results of the free-standing thin film, the attached thin film is modeled with ratio $t / d=0.1 .900$ grains with randomly assigned crystallographic orientation are contained in the substrate-attached thin film. To understand the influence of the substrate on the effective Young's modulus of the attached thin film, a parametric study is carried out for the substrate thickness and the substrate stiffness.

For the study of the substrate thickness, the ratio between the substrate thickness and the grain size, $h / d$, varies between 0.1 and 5 . For the study of the substrate stiffness, four types of material, namely polyimide, silicon, sapphire and polycrystalline diamond, are selected. This problem has been noted by Saha and Nix [9] in measuring the mechanical properties of the thin film attached to different substrate materials. Polyimide is a softest substrate with Young's modulus of 2.5GPa and Poisson's ratio of 0.34 ; while the polycrystalline diamond has the highest stiffness of 974GPa and Poisson's ratio of 0.10. The silicon substrate used is a piece of single crystal Si wafer with the (100) orientation. The corresponding elastic constants are $C_{11}=165.7 \mathrm{GPa}, C_{12}=63.9 \mathrm{GPa}$ and $C_{44}=79.6 \mathrm{GPa}$. Sapphire has Young's modulus of 470GPa and Poisson's ratio of 0.25 .

All the numerical results for the effective stiffness of the copper thin film attached to different substrate materials with diverse ratio $h / t$ are plotted in Fig. 9. Fig. 9 (a), (b), (c) and (d) are associated with one type of substrate material, namely polyimide, silicon, sapphire and polycrystalline diamond, respectively.

It is observed that the effective stiffness of the attached thin film varies dramatically when the ratio $h / t$ is less than 10 , and the value becomes stable after $h / t>$ 10. Another feature is that the effective modulus of the attached thin film increases with increasing substrate stiffness. When the stiffness of the substrate is lower than 
the stiffness of the film, the deformation of the grains in the film are not constrained by the substrate. Hence, the effective Young's modulus for the thin film attached on the polyimide substrate is close to the equivalent value of the free-standing thin film. As the stiffness of the substrate increases, more constraint on the deformation of the grains is imposed by the substrate. The effective modulus of the film therefore increases. Even when the substrate stiffness is comparable with the stiffness of the film material, for example, a copper thin film attached to a silicon substrate, the deformation of the grains in the film cannot be completely restrained by the substrate. The effective Young's modulus is not close to the Voigt upper bound. In the last case of polycrystalline diamond substrate which stiffness is seven times of that of copper bulk material, shown in Fig 9(d), the deformation of each grain in the film is strongly constrained by the substrate. Thus, each grain may be regarded as in parallel connection like Voigt's assumption. This leads to a higher stiffness than those obtained for the three-dimensional bulk material and the free-standing thin film.

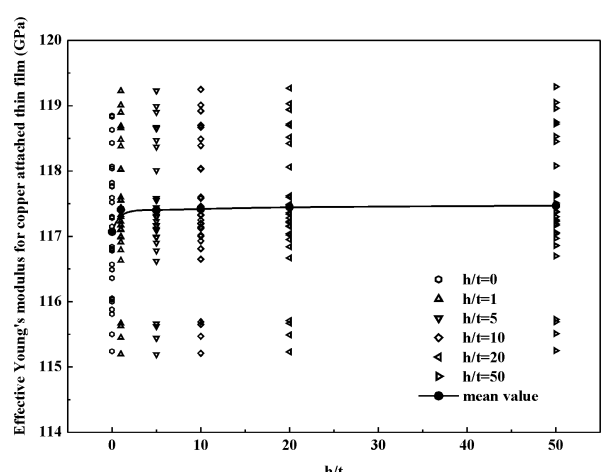

(a)

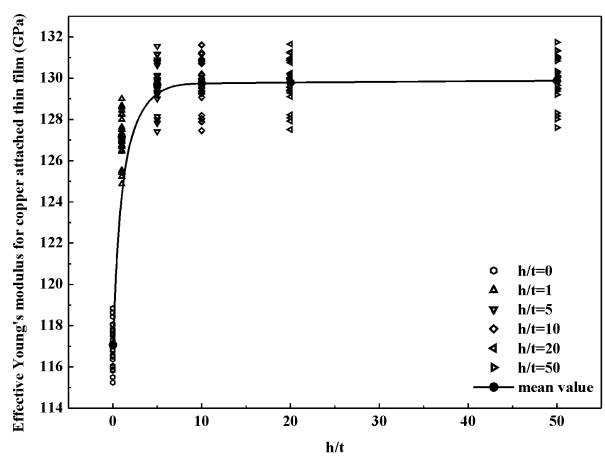

(c)

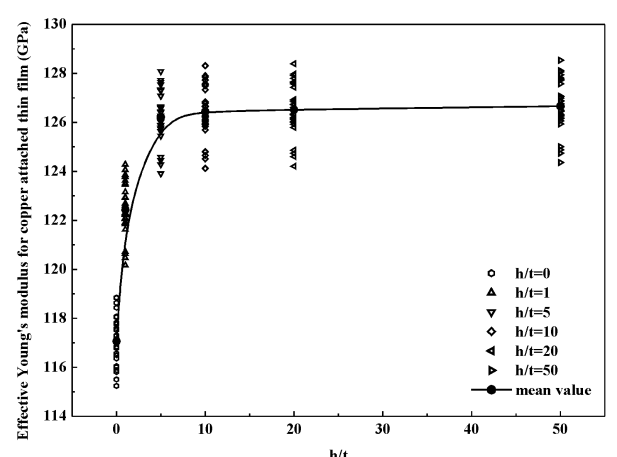

(b)

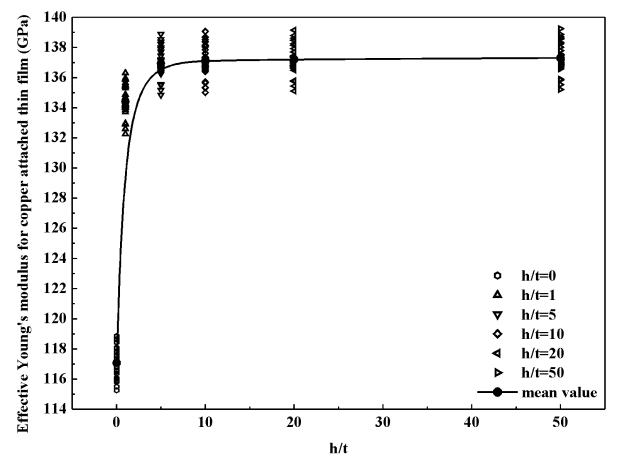

(d)

Figure 9. Scatter of the effective Young' s modulus for the copper thin film attached on different substrate materials. The ratio $h / t$ takes the value from 0 to 50 . The case, $h / t=0$, refers to the free-standing thin film with ratio $t / d=0$. 1 . (a) Polyimide; (b) Silicon; (c) Sapphire; (d) Polycrystalline diamond. 


\subsection{Summary of the numerical results}

In order to have a clear picture of the modulus difference in different geometry configurations, the effective stiffness of the thin film normalized by the corresponding value of copper bulk material $(127 \mathrm{GPa})$ are plotted as a function of the ratio $h / t$ in Fig. 10. From the lowest value of the stiffness in the free-standing configuration to the highest value in the configuration of a copper film attached to a diamond substrate, we see a difference in the order of $20 \%$.

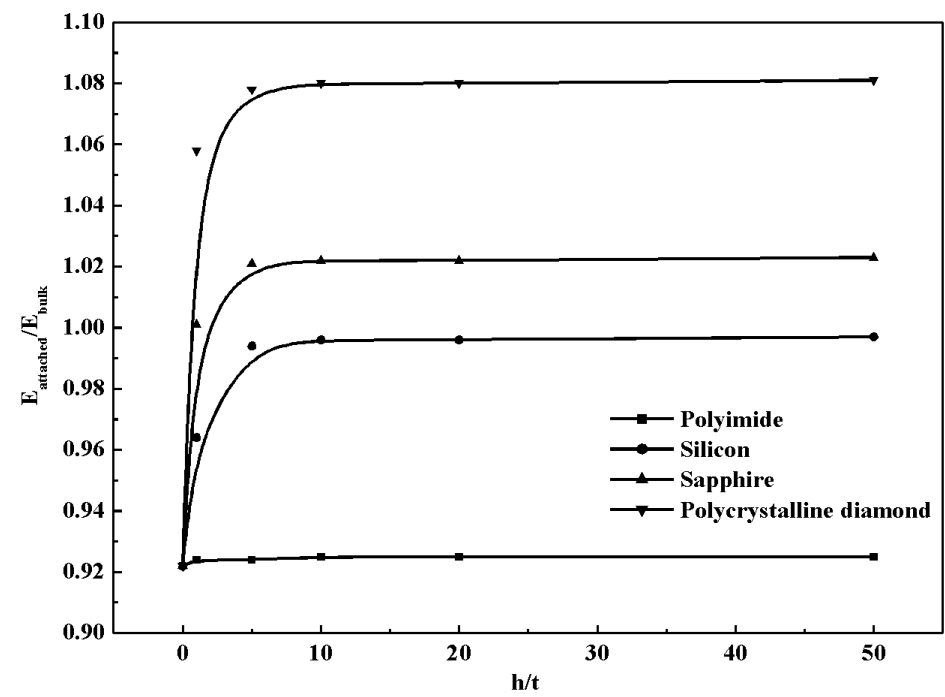

Figure 10. The normalized effective Young's modulus of the attached thin film ( $\left.E_{\text {attached }}\right)$ by the value of the bulk counterpart $\left(E_{\text {bulk }}\right)$ versus the ratio $h / t$ for different substrate materials. The case, $h / t=0$, is corresponding to the copper free-standing thin film.

With all the numerical results in our mind, we should have a better understanding of the modulus difference for polycrystalline aggregates in different configurations. The effective modulus of the thin film attached to a substrate is determined by the relative stiffness of the substrate to the film. The corresponding stiffness can range from the value of the free-standing film all the way to the upper bound (144 GPa) if the substrate is "rigid". Of course, in the real world, the diamond is the hardest material. Therefore, the effective modulus of $137 \mathrm{GPa}$ for the copper thin films may be the highest value that we may encounter in the real measurement. 


\section{Conclusions}

We use a finite element scheme with random distribution strategy to model the microstructure of polycrystalline aggregates. The accuracy and feasibility of this scheme is validated by comparing the numerical results for copper bulk material with the analytical solutions. This scheme is then applied to analyze the effective stiffness of the free-standing thin film and the substrate-attached thin film, respectively. The numerical results show that the effective stiffness of the free-standing thin film decreases with reducing the film thickness. And, the effective modulus of the substrate-attached thin film is affected by the stiffness ratio between the substrate and the film material. The spread of the effective Young's modulus in different configurations could be as large as $20 \%$.

In all our numerical simulation, no defects (such as micro-voids, grain boundary sliding, dislocations and so on) and size effect are involved. We may conclude that the geometric configurations do have certain influence on the effective modulus of a polycrystalline aggregate. 


\section{Appendix A. Elastic anisotropy}

In general, the elasticity of single crystal is anisotropic and described by a fourth-order elasticity tensor $\left(C_{i j k l}\right)$ or compliance tensor $\left(S_{i j k l}\right)$ with 21 independent material constants. The number of independent material constants depends further on the degree of crystal symmetry. Particularly, for the face-centered cubic (FCC) and body-centered cubic (BCC) crystal symmetry, the number of independent material constants reduces from 21 to 3 . Sometimes, it is convenient to express the elasticity and compliance tensors in matrix notation. For FCC or BCC crystals, the elasticity and compliance matrices are expressed as

$$
\begin{aligned}
& {\left[C_{I J}\right]=\left[\begin{array}{cccccc}
c_{11} & c_{12} & c_{12} & 0 & 0 & 0 \\
c_{12} & c_{11} & c_{12} & 0 & 0 & 0 \\
c_{12} & c_{12} & c_{11} & 0 & 0 & 0 \\
0 & 0 & 0 & c_{44} & 0 & 0 \\
0 & 0 & 0 & 0 & c_{44} & 0 \\
0 & 0 & 0 & 0 & 0 & c_{44}
\end{array}\right]} \\
& {\left[S_{I J}\right]=\left[\begin{array}{ccccccc}
s_{11} & s_{12} & s_{12} & 0 & 0 & 0 \\
s_{12} & s_{11} & s_{12} & 0 & 0 & 0 \\
s_{12} & s_{12} & s_{11} & 0 & 0 & 0 \\
0 & 0 & 0 & s_{44} & 0 & 0 \\
0 & 0 & 0 & 0 & s_{44} & 0 \\
0 & 0 & 0 & 0 & 0 & s_{44}
\end{array}\right]}
\end{aligned}
$$

where

$$
s_{11}=\frac{c_{11}+c_{12}}{\left(c_{11}-c_{12}\right)\left(c_{11}+2 c_{12}\right)}, s_{12}=\frac{-c_{12}}{\left(c_{11}-c_{12}\right)\left(c_{11}+2 c_{12}\right)}, \quad \text { and } \quad s_{44}=\frac{1}{c_{44}}
$$

Conversion from the tensor notation to the matrix notation is carried out according to the rules as follows.

$$
C_{1111} \rightarrow c_{11}, C_{1122} \rightarrow c_{12}, C_{1223} \rightarrow c_{64} \text {, and so on. }
$$


Note that the above independent material constants are given in the material coordinate system. When the crystal material coordinate does not coincide with a reference system (such as ANSYS global structure coordinate system), the elastic constants for the crystal in terms of global structure coordinate system are determined by

$$
\begin{gathered}
C_{i j k l}^{(S)}=\sum_{m=1}^{3} \sum_{n=1}^{3} \sum_{p=1}^{3} \sum_{q=1}^{3} t_{i m} t_{j n} t_{k p} t_{l q} C_{m n p q}^{(M)} \\
S_{i j k l}^{(S)}=\sum_{m=1}^{3} \sum_{n=1}^{3} \sum_{p=1}^{3} \sum_{q=1}^{3} t_{i m} t_{j n} t_{k p} t_{l q} S_{m n p q}^{(M)}
\end{gathered}
$$

where the superscript $(M)$ stands for the material coordinate system and the superscript $(S)$ represents the structural coordinate system, respectively. $t_{i j}$ are the components of transformation matrix (T) which is calculated in terms of Euler angles $(\alpha, \beta, \gamma)$ as

$$
\left(t_{i j}\right)=\left[\begin{array}{ccc}
\cos \alpha \cos \beta \cos \gamma-\sin \alpha \sin \gamma & -\cos \alpha \cos \beta \sin \gamma-\sin \alpha \cos \gamma & \cos \alpha \sin \beta \\
\sin \alpha \cos \beta \cos \gamma+\cos \alpha \sin \gamma & -\sin \alpha \cos \beta \sin \gamma+\cos \alpha \cos \gamma & \sin \alpha \sin \beta \\
-\sin \beta \cos \gamma & \sin \beta \sin \gamma & \cos \beta
\end{array}\right]
$$

where $\alpha \in[0,2 \pi], \beta \in[0, \pi]$ and $\gamma \in[0,2 \pi]$. In our study, we use sets of Euler angles $(\alpha, \beta, \gamma)$ to define the crystallographic orientation. Euler angles correspond to angular rotations that transform the global coordinate system to crystal material coordinate. 
Appendix B. Analytical predictions for the effective constants of polycrystalline aggregates

\section{B.1 Voigt-Reuss bounds}

With assumption of uniform strain for each grain throughout the whole polycrystalline body, Voigt approximations for the effective shear modulus $\left(G_{V}\right)$ and bulk modulus $\left(K_{V}\right)$ are determined by respectively [41]

$$
\begin{aligned}
& G_{V}=\frac{1}{8 \pi^{2}} \int_{0}^{2 \pi} d \alpha \int_{0}^{\pi} \sin \beta d \beta \int_{0}^{2 \pi} C_{1212} d \gamma \\
& K_{V}=\frac{C_{i i j j}}{9}
\end{aligned}
$$

On the other hand, Reuss assumed the uniform stress existing in each of the grains and derived the approximations for the effective shear modulus $\left(G_{R}\right)$ and bulk modulus $\left(K_{R}\right)$ as respectively [40]

$$
\begin{aligned}
G_{R} & =\left[\frac{1}{8 \pi^{2}} \int_{0}^{2 \pi} d \alpha \int_{0}^{\pi} \sin \beta d \beta \int_{0}^{2 \pi} S_{1212} d \gamma\right]^{-1} \\
K_{R} & =\frac{1}{S_{i i j j}}=\frac{C_{i i j j}}{9}
\end{aligned}
$$

For the FCC or BCC cubic crystal, they are given respectively by

$$
\begin{aligned}
& K_{R}=K_{V}=\frac{1}{3}\left(c_{11}+2 c_{12}\right) \\
& 5 G_{V}=\left(c_{11}-c_{12}\right)+3 c_{44} \\
& 5 / G_{R}=4\left(s_{11}-s_{12}\right)+3 s_{44}
\end{aligned}
$$

It was credited to Hill [21] who proved that Voigt approximation is the upper bound and Ruess approximation is the lower bounds, i.e. 


$$
K_{R} \leq K \leq K_{V}
$$

and

$$
G_{R} \leq G \leq G_{V}
$$

where subscript ' $R$ ' refers to Reuss lower bound and ' $V$ ' stands for Voigt upper bound. Meanwhile, the lower and upper bounds for the effective Young's modulus were given by Hill [21]

$$
E_{R} \leq E^{*} \leq E_{V}
$$

where

$$
\begin{aligned}
& \frac{1}{E_{R}}=\frac{1}{3 G_{R}}+\frac{1}{9 K_{R}} \\
& \frac{1}{E_{V}}=\frac{1}{3 G_{V}}+\frac{1}{9 K_{V}}
\end{aligned}
$$

\section{B.2 Hill's average}

Hill [21] recommended that the average of Reuss lower bound and Voigt upper bound may be a good approximation of the effective elastic constants for polycrystalline aggregates, that is

$$
\begin{aligned}
& K_{H}=\left(K_{R}+K_{V}\right) / 2 \\
& G_{H}=\left(G_{R}+G_{V}\right) / 2
\end{aligned}
$$

The shear modulus and bulk modulus connect with Young's modulus and Poisson's ratio by

$$
\begin{aligned}
& \frac{1}{E_{H}}=\frac{1}{3 G_{H}}+\frac{1}{9 K_{H}} \\
& v=\frac{1}{2}\left[1-\frac{3 G_{H}}{3 K_{H}+G_{H}}\right]
\end{aligned}
$$




\section{References:}

[1] D.A. Hardwick, Thin Solid Films.154 (1987) 109-124.

[2] W.D. Nix, Metall. Trans. A. 20 (1989) 2217-2245.

[3] R.P. Vinci and J.J. Vlassak, Annu. Rev. Mater. Sci. 26 (1996) 431-462.

[4] E. Arzt, Acta Mater. 46 (1998) 5611-5626.

[5] B. Freund and S. Suresh, Thin film materials: stress, defect formation, and surface evolution, Cambridge University Press, 2003.

[6] T.P. Weihs, S. Hong, J.C. Bravman, W.D. Nix, J. Mater. Res. 3 (1988) 931-942.

[7] H.B. Huang and F. Spaepen, Acta Mater. 48 (2000) 3261-3269.

[8] Y. Xiang, T.Y. Tsui, J.J. Vlassak, J. Mater. Res. 21(2006), 1607-1618.

[9] R. Saha and W.D. Nix, Acta Mater. 50 (2002) 23-38.

[10] J.N. Florando and W.D. Nix, J. Mech. Phys. Solids. 53 (2005) 619-638.

[11] D.Y.W. Yu and F. Spaepen, J. Appl. Phys. 95 (2004) 2991-2997.

[12] A.A. Volinsky, J. Vella, I.S. Adhihetty, V. Sarihan, L. Mercado, H.H. Yeung, W.W. Gerberic, Mat. Res. Soc. Symp. 649 (2001) Q5.3.1-Q5.3.5.

[13] H.D. Espinosa, B.C. Prorok and B. Peng, J. Mech. Phys. Solids. 52 (2004) 667-689.

[14] Y. Xiang, Ph. D. Dissertation, Harvard University, 2005.

[15] R.L. Mullen, R. Ballarini, Y. Yin, A.H. Heuer, Acta Mater. 45 (1997) 2247-2255.

[16] J.M.J. den Tooder, J.A.W. van Dommenlen, F.P.T.B. Aaijens, Modeling Simul. Mater. Sci. Eng. 7 (1999) 909-928.

[17] S. Weyer, A. Fröhlich, H. Riesch-Oppermann, L. Cizelj, Eng. Fract. Mech. 69 (2002) 945-958. 
[18] J. Choi, Ph. D. Dissertation, The Ohio State University, 2004.

[19] K.Y. Sze and N. Sheng, Finite Elem. Anal. Des. 42 (2005) 107-129.

[20] H.T. Zhang and C.T. Sun, Int. J. Mech. Sci. 48 (2006) 899-906.

[21] R. Hill, Proc. Phys. Soc. A65 (1952) 349-354.

[22] Z. Hashin and S. Shtrikman, J. Mech. Phys. Solids. 10 (1962) 343-352.

[23] L.J. Walpole, J. Mech. Phys. Solids. 14 (1966) 289-301.

[24] M.J. Beran, T.A. Mason, B.L. Adams, T. Olsen, J. Mech. Phys. Solids. 44 (1996) 1543-1563.

[25] R.M. Christensen, Mechanics of Composite Materials, John Wiley \& Sons, New York, 1979.

[26] P.R. Morris, Int. J. Eng. Sci. 8 (1970) 49-61.

[27] J.R. Willis, Adv. Appl. Mech. 21 (1981) 1-78.

[28] J.G. Berryman, J. Mech. Phys. Solids. 53 (2005) 2141-2173.

[29] K.W. Andrews, J. Phys. D: Appl. Phys. 11 (1978) 2527-2534.

[30] W. Pabst, G. Ticha, E. Gregorova, Ceram. Silik. 48(2004), 41-48.

[31] ANSYS10.0, ANSYS Inc., 2004.

[32] S. Kumar, Ph. D. Dissertation, The Pennsylvania State University, 1992.

[33] F. Barbe, L. Decker, D. Jeulin, G. Cailletaud, Int. J. Plast. 17 (2001a) 513-536.

[34] F. Barbe, L. Decker, G. Cailletaud, Int. J. Plast. 17 (2001b), 537-563.

[35] Y.W. Zhao and R. Tryon, Comput. Methods Appl. Mech. Engrg. 193 (2004) 3919-3934. 
[36] Y. Bhandari, S. Sarkar, M. Groeber, M.D. Uchic, D.M. Dimiduk, S. Ghosh, Comput. Mater. Sci. 41(2007) 222-235.

[37] W. Mendenhall, R.J. Beaver, B.M. Beaver, Introduction to Probability and Statistics, twelfth ed., Thomson, Singapore, 2006.

[38] H. Nozaki, M. Taya, ASME J. Appl. Mech. 68 (2001) 441-451.

[39] Cambridge Engineering Material Selector, Version 6, (2006).

[40] T. Mura, Micromechanics of Defects in Solids, second ed., Martinus, Netherlands, 1987. 